\title{
Interactive remote museum visits for older adults: an evaluation of feelings of presence, social closeness, engagement, and enjoyment in an social visit
}

\author{
Galena Pisoni \\ University of Trento \\ galena.pisoni@unitn.it
}

\author{
Florian Daniel \\ Politecnico di Milano \\ florian.daniel@polimi.it
}

\author{
Fabio Casati \\ University of Trento \\ fabio.casati@unitn.it
}

\author{
Charles Callaway \\ FBK-IRST \\ c.callaway@fbk.eu
}

\author{
Oliviero Stock \\ FBK-IRST \\ stock@fbk.eu
}

\begin{abstract}
This paper explores whether older adults can remotely participate in museum visits with the help of virtual environments. We design and build a system that supports shared museum co-visits between onsite visitors inside the museum and older adults from a care home. We make the experience more engaging by providing a meaningful story, connecting the objects in the museum. The aim of the study is to understand whether older adults are able to use such technology and to study the mediated sense of spatial presence, the experienced social closeness, and the level of participants' engagement and enjoyment in the visit. We discuss the relationship between these aspects and factors leading to a better remote experience for older adults. The results show that older adults enjoy and engage in remote visits, and that there is a positive correlation between enjoyment, engagement and social closeness.
\end{abstract}

Index Terms-Remote Participation, Older Adults, Museums, Interactive Visits, Drama-based approach

\section{INTRODUCTION}

In research on ageing much effort has been devoted to identifying viable alternatives for meetings and interactions for seniors physically separated from their homes, families and friends. Mobility constraints, bereavement, declining social networks or physical relocation have been proven to be closely associated with poor mental and physical health [1]. Staying in touch with people is important to older adults for social support, and active participation in social events reduces the risk of depression, anxiety and cognitive impairment [2]. Engaging in leisure activities has been shown to have positive effects on quality of life and general well-being [3].

In this paper we propose a new method that enables older adults to take part in a museum visit for a small group via drama and evaluation of its use. In this drama-based visit, the remote participant joins the onsite visitors as they walk around the museum with the help of technology, both parties are connected via an audio link, and the information about the objects are presented in the form of a story / drama connecting the objects in the exhibition. We test our method in situ, and our main objective is getting general feedback about remote visiting for older adults with a drama-based approach along with potential improvements on the overall user experience. Qualitative research methods (observations, semi-structured interviews, and content analysis) together with quantitative research methods (statistical analysis of data gathered from standardised questionnaires) are used as a means to better understand users' perceptions and their willingness to use technology. This work builds on our previous works in which we investigated what museum visitors share with non-visitors [4], and under which conditions older adults could join in museum visits remotely [5], [6].

The paper is strcutured as follows: we begin by describing the technology we use, we proceed with explaining the research questions of interest, the experimental setup and measures used, and we present the evaluation results. We conclude by discussing the current experimental findings and future directions.

\section{THE DRAMA APPROACH}

\section{A. Design stage}

We designed and developed our method through gathering input from the institutions involved in the project, investigating how to present content together with people who are familiar with needs of older adults, and incorporating good UI designing practices for older adults. The first author had two interviews with an educator and a psychologist (in a care home and a day centre respectively) familiar with the final aim of the project and its scope. With respect to following requirements for interface development, we based our work on interface design requirements for ICTs for older adults as described in previous research [7].

\section{B. Implementation of the system}

We designed the system that allows older adults to remotely and virtually join a group that is physically touring the museum. The central server hosts two types of servers: a positioning server, responsible for tracking the general location of each individual onsite visitor inside the museum with the help of iBeacons carefully located next to objects, and a VoIP (audio) server: responsible for establishing the audio channel between the onsite and remote participants. We developed two interventions, that is two software applications, one for the onsite visitors (a native iOS application) and one for the remote participants (a web application in a browser). The server additionally hosts the backend (or the APIs) serving both of the applications. All data exchanges and interactions leverage the Internet as a communication channel and are 


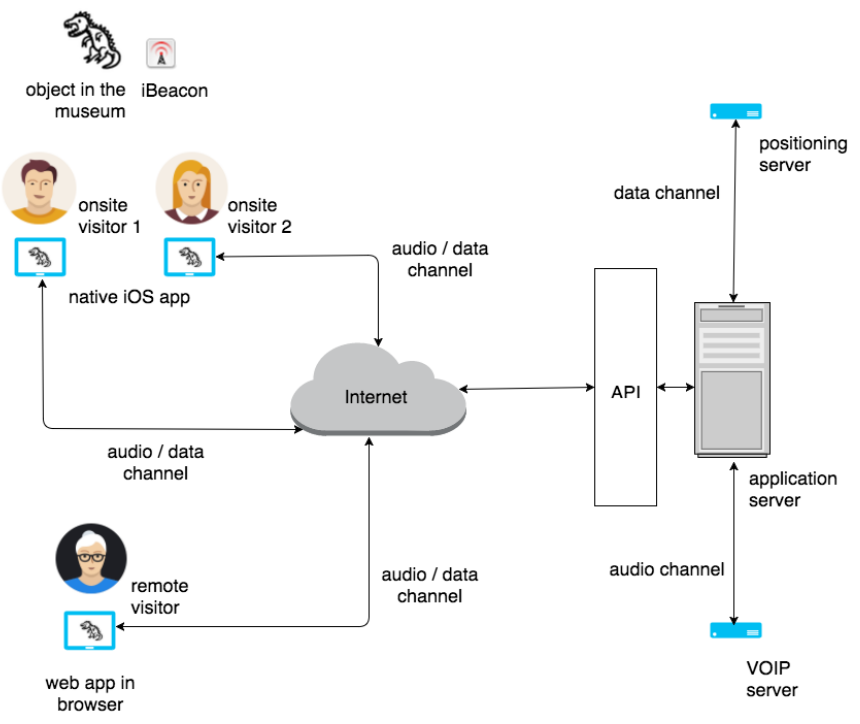

Fig. 1. The museum placed iBeacons at important exhibits. The position of the onsite visitor was determined via the beacons and the same content was delivered to the remote visitor at home. There was an audio channel between the remote and onsite visitor.

mediated by a centralized application server. The architecture of the approach is illustrated in Figure 1.

The system tracks visitors' locations inside the museum and presents visitors with relevant presentations as they arrive at an object. The system synchronously delivers the same digital content to the remote participant: each second the application server receives sensor updates from all clients, and then redistributes the combined information to all clients, informing them of the position, microphone, and other sensor data from each onsite client. When the onsite visitors reach a new position, the picture they see at the top of the interface is updated to reflect this change. The onsite visitors use the onsite application, while the remote visitor(s) at the same time use the remote application inside the care home and are able to follow one of the onsite visitors as they move inside the museum and to engage in conversations with everyone via the audio channel.

The system incorporates an audio drama (written and recorded by a professional dramaturge and recording studio), written specifically for various objects in the museum. Selected exhibits on the path are associated with specific story segments. The story segments are delivered to both onsite and remote visitor(s) only when all the onsite visitors are close to the triggering exhibit's iBeacon and when the group is not talking (so as to avoid interrupting a conversation). The APIs determine the time when a story segment should play, or when the system is in a "conversation phase", i.e a one minute period after a story segment has played, and delivers this information to all the clients. In this phase the screen is black and participants can discuss further the presented content without interruption. Several connectors, i.e. short phrases that support and inspire discussion are played, to encourage conversation if there's a lack of ideas of what to talk about.
The story is about two aliens landing close to the museum, who are not familiar with the history of the planet Earth. They learn more about evolution on Earth based on the exhibits in the museum. The drama is written in a non-linear fashion allowing the onsite group to choose their own path. The story ends with aliens discovering an important insight that may save their race from extinction.

\section{The remote application}

The interface of the remote application (Figure 2) is in the form of a theatre stage. The "journey" to the museum starts with a small animation representing the opening of the red curtains on the stage. Once the user selects which onsite visitor to follow, the application does not require any further interaction in keeping with the suggestions from related work. The theatre background is automatically updated based on the exhibit in front of which the selected person in the museum is actually standing, and the remote participant can see the object the group members are currently looking at. In order to facilitate and stimulate conversation, when the system is not playing drama, the onsite visitors are depicted facing the remote participant. While the audio drama is playing and the participants are following the story, the onsite visitors are depicted facing towards the presented exhibit.

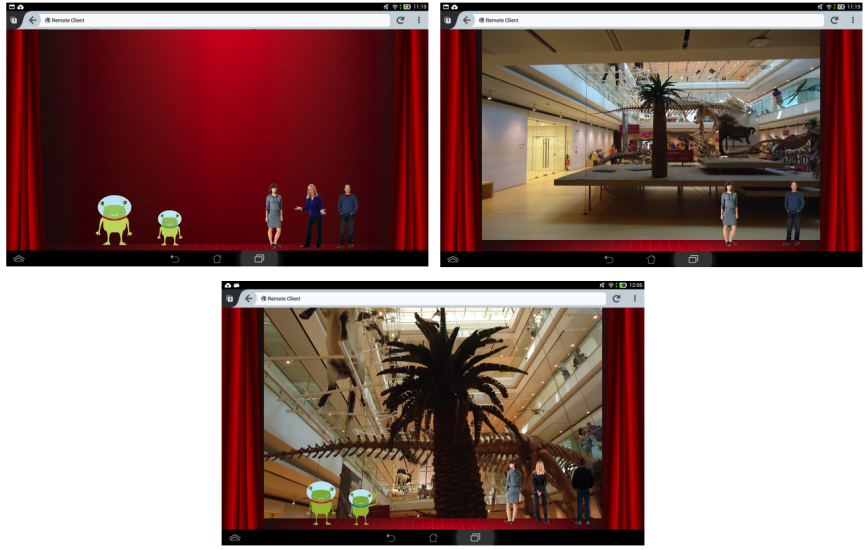

Fig. 2. Remote visiting application: all the onsite companions are presented at the stage, and the remote participant has the option to choose who to follow in the visit (a), after the older adults selects the person to follow, the background is automatically updated with a picture of the object that the selected onsite visitor is seeing (b), and when all the onsite companions are at the same object, and there is a story segment associated with this object, the system enters in drama state, the group starts to listen to a story segment, the onsite participants are facing the object and there are aliens on the stage, telling the story of the object.

\section{RESEARCH QUESTIONS}

Our research questions are devised to test the drama approach, and our research focuses specifically on the remote participant(s) in our approach. Data regarding the onsite visitors were collected and analyzed, but mainly in order to examine their influence on the remote visitor experience.

RQ1: Is a drama-based visit an enjoyable experience for the older adult? 
RQ2: Do older adults feel engaged during a drama-based remote visit?

RQ3: Do older adults experience spatial presence in a dramabased remote visit?

RQ4: Do remote participants experience social closeness with the rest of the group in a drama-based visit?

RQ5: Do the dynamics of the onsite group influence the experience of the remote participant(s)?

\section{METhOD}

\section{A. Participants}

We had two types of participants: remote visitor(s) and onsite visitor(s).

Older adult participants were selected from a care home far away from the museum, at a home for many older adults with physical difficulties in moving. Older adults were screened based on their functional cognitive level. Short Portable Mental Status Questionnaire (SPMSQ) [8] is a short questionnaire assessing levels of intellectual functioning for older adults: a score between 0 and 2 corresponds to intact intellectual functioning, 3 to 4 indicates mild intellectual impairment, 5 to 7 corresponds to moderate intellectual impairment and 8 to 10 indicates severe intellectual impairment. We wanted to investigate whether our tool can be used and is appropriate for older adults with diverse abilities, so we only excluded participants with severe intellectual impairment and for the purpose of the experiment we chose participants with an SPMSQ score below 7.

We recruited onsite younger adult visitors by word of mouth inside the care home. The younger adults participated in the study from the museum. Each session lasted around 45 minutes and we had 15 older adults that showed interest in participating. Three participants did not take part: 2 due to audio problems at the scheduled session and 1 did not feel like participating that day, which left us with 12 participants in total.

\section{B. Measures}

We used the following measures for our study.

1) Engagement: The ITC-Sense of Presence Inventory (ICT-SOPI) questionnaire [9] is a technology-agnostic presence scale that consists of 46-items, each validated for independent use. We used the Engagement sub-scale (e.g., "I felt involved (in the displayed environment)", "I enjoyed myself", "My experience was intense").

2) Spatial presence: We also used the ICT-SOPI questionnaire here, using the Sense of Physical Space sub-scale measuring the sense of being located in another place, in this case the museum (e.g., "I had a sense of being in the scenes displayed", "I felt I could have reached out and touched things (in the displayed environment)").

3) Social closeness: The Inclusion of Other in Self scale (IOS) [10] is a single-item, graphical measure that shows two circles for self and others at various levels of distance until they substantially overlap. Our participants indicated which one best represents the perceived interaction with the onsite companions.

4) Enjoyment: Four items on a five-point Likert scale from the GEQ scale [11] measured enjoyment in the experience (e.g., "I enjoyed the experience").

5) Onsite group data: We collected the following data regarding the onsite visitors:

1) The position of each onsite member each second

2) The state of the system at each second, i.e. in drama, observe or just visiting

3) Audio recordings of the sessions

\section{Data Collection}

All the scales of the independent measures were combined into one questionnaire that we administered to each participant when they finished the testing. We additionally had a semistructured interview, debriefing the participants if they liked the experience, which drama piece was their favorite and least favorite, what they would change, and if they had any additional comments about either the characters or the interface.

\section{Results}

The three participants who did not finish the study and did not complete the questionnaire were omitted from data analysis, so our final data set contains 12 participants. Data analysis was performed with the $\mathrm{R}$ statistical package.

We calculated Cronbach's Alpha of the constructs to test if the items measure the same construct, and to measure the internal consistency. The reliability of the 4 scales that were used in the questionnaires is acceptable (Cronbach's Alpha $>0.4$ ), therefore, the items belonging to each scale seem to describe one construct and all scales can be calculated as mean scores. Taking into account that Social Closeness was measured only by one item, we could not calculate Cronbach's Alpha for it. We report on the means and standard deviation of each construct in (Figure 3).

Through ANOVA, we tested whether age had any significant effects on our dependent variables, but no correlation was found.

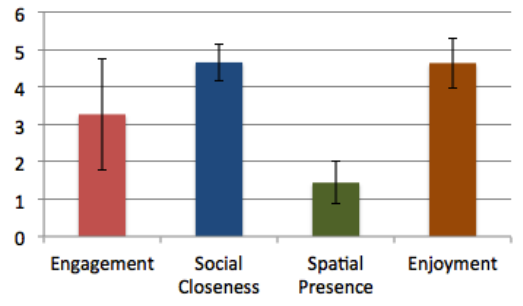

Fig. 3. Means and standard deviations of the constructs measured in the study.

Next we looked at the correlations between independent variables. We found that Enjoyment correlates significantly with Engagement $(\mathrm{r}(12)=0.834, \mathrm{p}<0.001)$ and Social Closeness $(\mathrm{r}(12)=0.605, \mathrm{p}<0.05)$. Also Social Closeness correlated 
with Engagement $(\mathrm{r}(12)=0.605, \mathrm{p}<0.05)$ and Enjoyment $(\mathrm{r}(12)=0.594, \mathrm{p}<0.05)$ but not with Spatial presence. Furthermore, Engagement correlates significantly with Spatial Presence $(\mathrm{r}(12)=0.660, \mathrm{p}<0.05)$ and Enjoyment correlates significantly with Spatial Presence $(\mathrm{r}(12)=0.622, \mathrm{p}<0.05)$. What we understood from this is that within the use of our tool, the constructs of Engagement, Enjoyment and Social Closeness are highly correlated, whereas the constructs of Social Closeness and Spatial Presence don't seem to correlate.

We discuss the results further (together with the observation data) according to our five research questions outlined previously.

1) RQ1: Is a drama-based visit an enjoyable experience for the older adult?: In general we found a high degree of enjoyment for the participants. Participants liked the visit and they found it fun. Our non traditional alien "guides" in the museum visit attracted attention to our participants, and each session started with the elderly curiously questioning what and who they are. The interactions between visitors (remote and onsite) indicated a high level of overall enjoyment, allowing users to focus on both looking at the exhibits and talking to each other.

Some of the participants suggested having videos instead of static photos, or perhaps a slideshow of several photos changing over time to follow the story flow.

2) RQ2: Do older adults engage in a drama-based remote visit?: Participants expressed comments and their points of view about the exhibits presented, commenting primarily on the content of the museum. The drama pieces, together with the pictures of the objects and the people onsite, allowed the elderly to deepen their interest in and develop curiosity for the exhibits that they saw.

The connectors that followed story segments sometimes stopped discussion rather than promote it. This was a drawback especially for those elderly that forgot what they wanted to say after the connector.

3) RQ3: Do older adults experience spatial presence in a drama-based remote visit?: Our intuition was not confirmed, and our study revealed that our system didn't score highly on the construct of spatial presence. Our participants didn't feel as though "they are there" in the displayed environment. We believe that the lack of continuous flow of the image (a limitation of the proximity-based positioning system we used) is the main cause for the lack of appreciation of the spatial rendering factor.

4) RQ4: Do remote participant experiences social closeness with the rest of the group in a drama-based visit?: The results show that the older adults felt as though they were with someone and the feeling of experiencing social closeness was quite high. In our experiment, people participated not only as individuals, but as part of a social group, going for a visit of a museum together. Being together with other people in the shared activity of visiting a museum created a more subjective feeling of group, and created a feeling of togetherness that was reflected byboth onsite and remote visitors in their questionnaire.
5) RQ5: Do the dynamics of the onsite group influence the experience of the remote participant?: The groups typically stayed together throughout the visit and there was usually a strong overall tendency for maintaining the coherence of the group. What we observed is that there was much more interaction and communication when the onsite group consisted of two people, and in general the experience was more fun as observed by the research assistants involved in the testing. We observed the same dynamic when two elderly adults participated together from the care home.

Limitations: The research presented in this paper has a number of limitations. The study involved 12 older adults which is a relatively small sample. A bigger sample is needed to understand if the findings discovered are generalisable to larger audiences.

\section{CONCLUSIONS}

In this experiment we explored whether older adults are able to remotely participate in museum visits from home, and explored further concepts such as engagement, enjoyment, and presence in a remote museum visit using virtual environments. We found evidence that remote participants feel as if they are part of a group visiting the museum, and that they engage in them and enjoy the virtual visits. Our museum visits proved to be highly interactive experiences in which the remote participant engages, but we understand from our results that this engagement can be attained only if they enjoy themselves and feel part of the group, and vice versa.

\section{REFERENCES}

[1] C. Garattini, J. Wherton, and D. Prendergast, "Linking the lonely: an exploration of a communication technology designed to support social interaction among older adults," Universal Access in the Information Society, vol. 11, no. 2, pp. 211-222, 2012.

[2] J. Golden, R. M. Conroy, and B. A. Lawlor, "Social support network structure in older people: underlying dimensions and association with psychological and physical health," Psychology, health \& medicine, vol. 14, no. 3, pp. 280-290, 2009.

[3] C. Cannuscio, J. Block, and I. Kawachi, "Social capital and successful aging: The role of senior housing," Annals of Internal Medicine, vol. 139, no. 5_Part_2, pp. 395-399, 2003.

[4] G. Kostoska, B. Valeri, M. Baez, F. Daniel, and F. Casati, "Collecting memories of the museum experience," in Proc. CHI'13, pp. 247-252.

[5] G. Kostoska, "Virtual, remote participation in museum visits by older adults: A feasibility study," in Proceedings of the 8th International Conference on Personalized Access to Cultural Heritage - PATCH'15.

[6] G. Kostoska, A. P. Vermeeren, J. Kort, and C. Gullström, "Videomediated participation in virtual museum tours for older adults," in 10th International Conference on Design \& Emotion, 27-30 September 2016.

[7] L. Muskens, R. van Lent, A. Vijfvinkel, P. van Cann, and S. Shahid, "Never too old to use a tablet: Designing tablet applications for the cognitively and physically impaired elderly," in Computers Helping People with Special Needs. Springer, 2014, pp. 391-398.

[8] E. Pfeiffer, "A short portable mental status questionnaire for the assessment of organic brain deficit in elderly patients," Journal of the American Geriatrics Society, vol. 23, no. 10, pp. 433-441, 1975.

[9] J. Lessiter, J. Freeman, E. Keogh, and J. Davidoff, "A cross-media presence questionnaire: The itc-sense of presence inventory," Presence, vol. 10 , no. 3, pp. 282-297, 2001.

[10] A. Aron, E. N. Aron, and D. Smollan, "Inclusion of other in the self scale and the structure of interpersonal closeness." Journal of personality and social psychology, vol. 63, no. 4, p. 596, 1992.

[11] Y. A. de Kort, W. A. IJsselsteijn, and K. Poels, "Digital games as social presence technology: Development of the social presence in gaming questionnaire (spgq)," Proceedings of PRESENCE, pp. 195-203, 2007. 